\title{
Quantum Cosmology of Quadratic $f(R)$ Theories with a FRW Metric
}

\author{
V. Vázquez-Báez ${ }^{1}$ and C. Ramírez ${ }^{2}$ \\ ${ }^{1}$ Facultad de Ingeniería, Benemérita Universidad Autónoma de Puebla, 72000 Puebla, PUE, Mexico \\ ${ }^{2}$ Facultad de Ciencias Físico Matemáticas, Benemérita Universidad Autónoma de Puebla, P.O. Box 165, 72000 Puebla, PUE, Mexico
}

Correspondence should be addressed to V. Vázquez-Báez; manuel.vazquez@correo.buap.mx

Received 1 April 2017; Accepted 17 May 2017; Published 18 June 2017

Academic Editor: Anzhong Wang

Copyright (C) 2017 V. Vázquez-Báez and C. Ramírez. This is an open access article distributed under the Creative Commons Attribution License, which permits unrestricted use, distribution, and reproduction in any medium, provided the original work is properly cited.

\begin{abstract}
We study the quantum cosmology of a quadratic $f(R)$ theory with a FRW metric, via one of its equivalent Horndeski type actions, where the dynamic of the scalar field is induced. The classical equations of motion and the Wheeler-DeWitt equation, in their exact versions, are solved numerically. There is a free parameter in the action from which two cases follow: inflation + exit and inflation alone. The numerical solution of the Wheeler-DeWitt equation depends strongly on the boundary conditions, which can be chosen so that the resulting wave function of the universe is normalizable and consistent with Hermitian operators.
\end{abstract}

\section{Introduction}

Since its formulation, general relativity has been a successful theory, verified in many ways and at any scale. However, there are instances where it does not reproduce in a precise way the results of observations, in particular the origin of the universe and the early and present inflationary phases. One way to describe these issues has been given by means of modified gravity $f(R)$ theories. Starobisnky [1] has proposed $f(R)$ theory as an effective action of gravity obtained by coupling quantum matter fields to gravity, which explains inflation and reheating, and recently it has been used to explain the effects of dark matter and dark energy $[2,3]$ (see also $[4,5])$. Even if these theories appear as effective theories, one appealing feature of them is that they are pure gravity theories, although they are higher order. However, under certain conditions, it is possible to give actions of Horndeski type equivalent to $f(R)$ actions $[6,7]$, which have the advantage of being second-order and consistent with the usual inflationary or dark energy scenarios where there are scalar fields coupled to Einstein relativity.

Another scenario where $f(R)$ theory has been considered is the origin of the universe by a tunneling mechanism from "nothing" to the de Sitter phase of Starobinski's model [8], where a description of the universe in the framework of quantum cosmology is given, from which the tunneling probability, the subsequent curvature fluctuations, and the duration of the inflationary phase were computed, in the WKB approximation. Quantum cosmology of $f(R)$ theories has been studied also in [9-12]. One frequent problem related to the wave function of the universe is its interpretation and, related to it, its normalizability, which might depend on the initial conditions [9].

In this work, we consider the quantum cosmology of $f(R)=R+\alpha R^{2}$ theory with the FRW metric in the formulation due to O'Hanlon [6], with an auxiliary scalar field $\phi$, and give a numerical solution for the Wheeler-DeWitt equation in its exact form. Considering that the scale factor $a$ is positive, we require that the wave function of the universe vanishes at $a=0$, in order for the conjugate momentum of $a$ to be hermitic. For the numerical computation, we consider a compact domain in $a$ and $\phi$. We get that the solution is consistent to zero on all boundaries, corresponding to normalizability. This feature seems to consistently persist in the noncompact limit. In the second section, we make a short analysis of the classical solutions and in the third section we show the numerical solution of the WdW equation considering values of the parameter $\alpha$ for which classically the solutions are qualitatively different. In the last section, we draw some conclusions. 


\section{Lagrangian Analysis}

Let us focus on the $f(R)$ model for gravity (see [4]) without matter which has an action given by $\left(1 / 2 \kappa^{2}\right) \int d t \sqrt{-g} f(R)$; variation with respect to the metric $g_{\mu \nu}$ results in

$\delta A$

$$
=\frac{1}{2 \kappa^{2}} \int \sqrt{-g}\left[\frac{1}{2} g^{\mu \nu} f(R)+\frac{\partial f(R)}{\partial R} \frac{\delta R}{\delta g_{\mu \nu}}\right] \delta g_{\mu \nu} d^{4} x,
$$

which leads to the following equations of motion:

$$
F(R) R_{\mu \nu}-\frac{1}{2} f(R) g_{\mu \nu}-\nabla_{\mu} \nabla_{\nu} F(R)+g_{\mu \nu} \square F(R)=0,
$$

where $F(R)=f^{\prime}(R)$.

Recalling that for a FRW geometry the metric tensor is the following:

$$
g_{\mu \nu}=\operatorname{diag}\left(-N^{2}, \frac{a^{2}}{1-k r^{2}}, a^{2} r^{2}, a^{2} r^{2} \operatorname{sen}^{2} \theta\right),
$$

then the scalar curvature is given by

$$
R=\frac{6}{a^{2}}\left(\frac{a \ddot{a}}{N^{2}}+\frac{\dot{a}^{2}}{N^{2}}-\frac{a \dot{a} \dot{N}}{N^{3}}+k\right)
$$

which written in terms of the Hubble factor becomes $R=$ $12 H^{2}+6 \dot{H}$ once we fixed the gauge field to $N=1$ and set $k=0$. With the scalar curvature as a function of $H$, as stated above, the field equations (2) are

$$
\begin{aligned}
3 F H^{2} & =\frac{1}{2}(F R-f)-3 H \dot{F}, \\
-2 F \dot{H} & =\ddot{F}-H \dot{F},
\end{aligned}
$$

where $F=F(H(t))$ is regarded as a scalar degree of freedom, the so-called "scalaron" $\varphi[1]$.

This approach presents some calculational advantages; for instance, it is evident that, treating $H$ and $\varphi$ as the dynamical fields, the above equations of motion are secondorder; choosing $a(t)$ as the dynamical variable leads to thirdorder equations; also, the dynamic of the scale factor is simply given by $a=\exp \left[\int H d t\right]$, provided one can solve for $H(t)$. Nevertheless, the previous statements are based on the one hand on setting $k=0$ and on the other hand on the fixing on the gauge $N=1$. Let us examine these two conditions.

For $k \neq 0$, the curvature in terms of $H$ reads $R=$ $12 H^{2}+6 \dot{H}+6 k / a^{2}$. Nevertheless, this loss of generality is not a problem since the observations favor an almost $(k \approx 0)$ flat universe [13]. On the other hand, the choice $N(t)=1$ for the gauge field on the Lagrangian formulation represents no difficulty; however, if we want to implement the Hamiltonian formulation of the theory and thus the quantum formulation, we have to keep the gauge arbitrary. Additionally, for the Hamiltonian formulation of an $f(R)$ theory, we should implement Ostrogradsky's formalism [14-16] to deal with the higher order derivatives in the Lagrangian; for instance, the terms containing derivatives of the lapse function must be integrated out of the action since, being a gauge field, it cannot have dynamics; this in turn produces third-order derivatives for the scale factor and in general it is not possible to eliminate all the derivatives of $N$ as can be seen in the following Lagrangian:

$$
\begin{aligned}
L= & -\frac{24 \alpha a \ddot{a}}{\dot{a}} N^{3}+\frac{12 \alpha a \ddot{a}^{2}}{N^{3}} \\
& +\alpha \dot{a}^{2}\left(-\frac{24 \ddot{a}}{N^{3}}+\frac{72 k}{a N}+\frac{36 a \dot{N}^{2}}{N^{5}}\right)+\frac{36 \alpha \dot{a}^{4}}{a N^{3}} \\
& +\frac{36 \alpha k^{2} N}{a}-\frac{6 a \dot{a}^{2}}{N}+6 k a N,
\end{aligned}
$$

which corresponds to the quadratic Starobinsky's model, $f(R)=R+\alpha R^{2}$. The third derivative of $a$ can be seen in the first term on the right; also, we can see the presence of a derivative of $N$, which complicates the Hamiltonian formulation. These complications can be avoided in Starobinsky's model, with the FRW metric plugged in an O'Hanlon type of action [6] and given by

$$
A=\frac{1}{2 \kappa^{2}} \int \sqrt{-g}[R+\phi(\beta \phi+R)],
$$

where $\beta$ is a free parameter. This Horndeski type action resembles the action used in [8], where the definition of the scalar curvature is regarded as a constraint and used to eliminate $\ddot{a}$ terms in the action.

A variation with respect to $\phi$ results in $\phi=-(1 / 2 \beta) R$ which leads to

$$
A=\frac{1}{2 \kappa^{2}} \int \sqrt{-g}\left(R-\frac{1}{4 \beta} R^{2}\right) .
$$

Thus, action (7) is completely equivalent to that of the Starobinsky's model. Once we enter the FRW metric into this action, we are able to partially integrate the second-order derivative terms and all the derivatives of $N$ unlike the case mentioned above; thus, we get

$$
\begin{aligned}
A & =\frac{1}{\kappa} \int d t\left[3(1+\phi)\left(N a k-N^{-1} a \dot{a}^{2}\right)-3 N^{-1} a^{2} \dot{a} \dot{\phi}\right. \\
& \left.+\frac{1}{2} \beta N a^{3} \phi^{2}\right] .
\end{aligned}
$$

After fixing the gauge $N=1$, the resulting equations of motion of $N, a$, and $\phi$, from this action, are

$$
\begin{aligned}
& \left(6 a \dot{a}^{2}+6 k a\right)(1+\phi)+6 a^{2} \dot{a} \dot{\phi}+\beta a^{3} \phi^{2}=0, \\
& \left(4 a \ddot{a}+2 \dot{a}^{2}+2 k\right)(1+\phi)+2 a^{2} \ddot{\phi}+4 a \dot{a} \dot{\phi}+\beta a^{2} \phi^{2} \\
& \quad=0, \\
& 3 a^{2} \ddot{a}+3 a \dot{a}^{2}+3 k a+\beta a^{3} \phi=0 .
\end{aligned}
$$

From (12), we get

$$
\phi=-\frac{3}{\beta a^{2}}\left(a \ddot{a}+\dot{a}^{2}+k\right),
$$




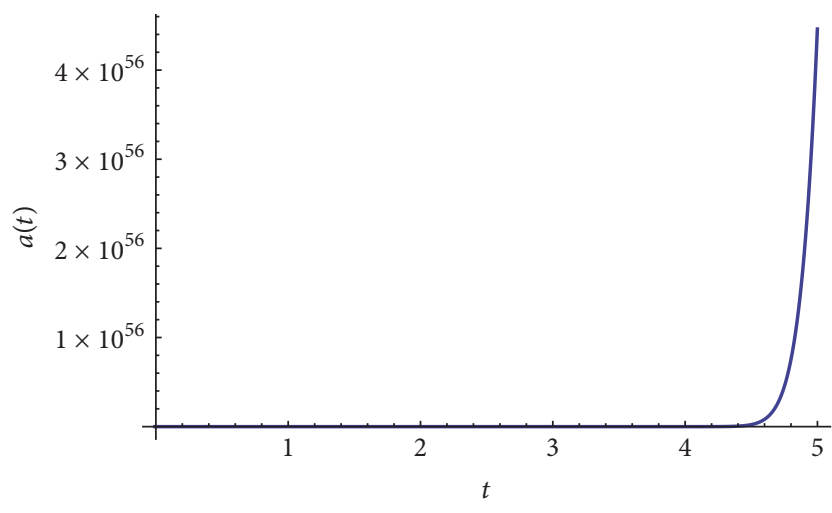

FIGURE 1: Numerical profile of $a(t)$ with initial conditions $a(0)=$ $10^{-20}, \dot{a}(0)=10^{-20}, \ddot{a}(0)=10^{-17}, \beta=-100$, and $k=0$.

which substituted in (10) gives

$$
\begin{aligned}
3 k^{2}+ & \dot{a}^{2}\left(6 a \ddot{a}-9 \dot{a}^{2}-2 \beta a^{2}-6 k\right) \\
& +a^{2}\left(6 \ddot{a} \ddot{a}-3 \ddot{a}^{2}-2 k \beta\right)=0 .
\end{aligned}
$$

Equation (11) is redundant. Obviously, (14) can be solved only numerically. We fix the parameter $\beta<0$ in order to produce a consistent profile for the evolution of the scale factor. In general, whatever the value of $\beta$, we have an inflation stage (see Figure 1), but for sufficiently large values, this inflationary phase becomes an exit, as we depict in Figure 2. Also, in this figure, we can see that as $a$ reaches a certain value, its evolution stops and the universe freezes out; this feature of the model should change in the presence of matter.

\section{Wheeler-DeWitt Equation}

In this section, we give the canonical quantum formulation of the previous cosmological model. The canonical momenta are

$$
\begin{aligned}
& \pi_{a}=-\left[\frac{12 a \phi \dot{a}}{N}+\frac{12 a \dot{a}}{N}+\frac{6 a^{2} \dot{\phi}}{N}\right], \\
& \pi_{\phi}=-\frac{6 a^{2} \dot{a}}{N}, \\
& \pi_{N}=0,
\end{aligned}
$$

from which we get the usual form of the Hamiltonian as a product of a first-class constraint times a Lagrange multiplier, $H=N H_{0}$, with

$$
H_{0}=-6 k a \phi-6 k a-\beta a^{3} \phi^{2}+\frac{\phi \pi_{\phi}^{2}}{6 a^{3}}+\frac{\pi_{\phi}^{2}}{6 a^{3}}-\frac{\pi_{a} \pi_{\phi}}{6 a^{2}}
$$

After quantization $\left(q \rightarrow \hat{q}, \pi \rightarrow-i \hbar \partial_{q}\right)$, the first-class constraint $H_{0}$ becomes a condition on the state wave function of the universe, the Wheeler-DeWitt equation $\widehat{H} \psi=0$.

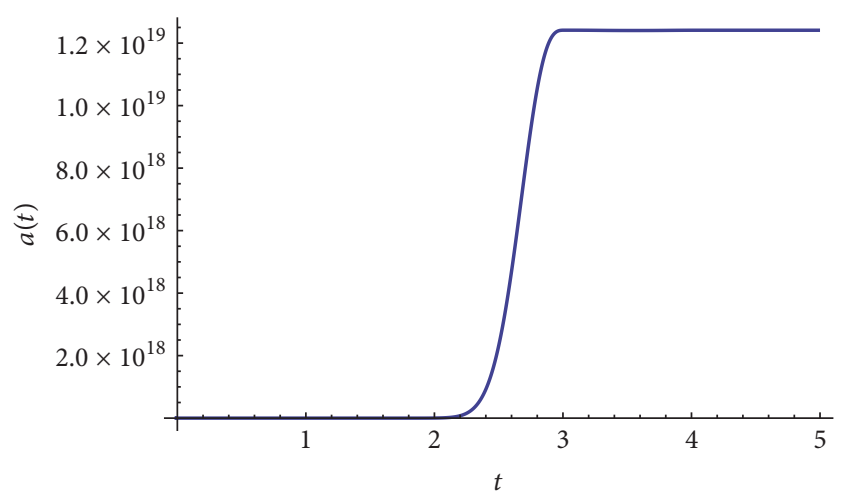

Figure 2: Numerical profile of $a(t)$ with initial conditions $a(0)=$ $10^{-20}, \dot{a}(0)=10^{-20}, \ddot{a}(0)=10^{-17}, \beta=-200$, and $k=0$.

We chose the Weyl ordering for the ambiguous operator products. Thus, we get the nonlinear equation

$$
\begin{gathered}
\widehat{H} \psi=\left[-\hbar^{2}(\phi+1) \frac{\partial^{2}}{\partial \phi^{2}}+a \hbar^{2} \frac{\partial^{2}}{\partial \phi \partial a}-2 \hbar^{2} \frac{\partial}{\partial \phi}\right. \\
\left.-6 \beta a^{6} \phi^{2}-36 k a^{4}(\phi+1)\right] \psi(a, \phi)=0 .
\end{gathered}
$$

This equation does not admit analytic solutions, unless approximation methods are considered [8]. In order to solve in the exact setting, we do it numerically in a compact domain $\left[0, a_{0}\right] \times\left[-\phi_{0}, \phi_{0}\right]$. The fact that $a \geq 0$ can spoil hermiticity of the conjugate momentum of $\pi_{a}$. In order to avoid this problem, we require that the wave function vanishes at $a=$ 0 [17]. As (17) is first-order in the derivatives of $a$ and second-order in the derivatives of $\phi$, it requires another two conditions in the boundaries in the $\phi$ direction, and we take them to be symmetric $\psi\left(a, \pm \phi_{0}\right)=f(a)$, where $f(a)$ is a suitable function. It turns out that conditions of the type $f(a)=0$ or $\partial_{\phi} \psi\left(a, \pm \phi_{0}\right)=0$ restrict too strong the wave function and lead to the numerical trivial solution. Otherwise, we see that the wave function is nonvanishing in a well-defined region of the domain, as shown in Figure 3. In fact, a careful inspection of this numerical solution shows that for a function $f(a)$ which vanishes at $a=0$ and $a=a_{0}$, for instance, $f(a)=\sin \left(\pi_{a} / a_{0}\right) / \phi_{0}$, and then the wave function vanishes at the boundaries $a=0$ and $a=a_{0}$ and at $\phi=$ $\pm \phi_{0}$ vanishes as $\phi_{0} \rightarrow \infty$, pointing to a normalizable wave function.

The normalizability of the wave function, despite being motivated by the numerical analysis, can be analytically justified if we consider the solution in the neighborhood of the boundary. In order to do it in the $a$-direction, we look for a series solution of the form

$$
\psi(a, \phi)=\sum_{n=0}^{\infty} f_{n}(\phi) a^{n}
$$




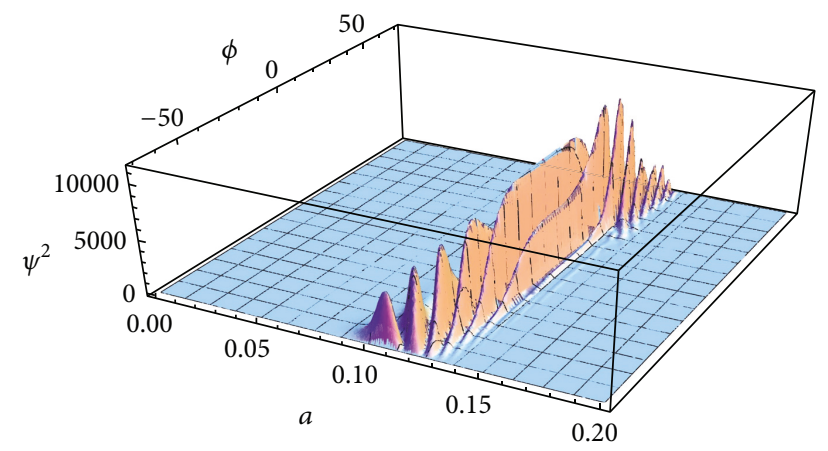

FIgURE 3: Numerical profile of $\psi(a, \phi)$ with initial conditions: $\psi(0, \phi)=0$ and $\psi\left(a, \phi_{0}\right)=f(a)$, where $\phi_{0}$ defines half the width of our solution domain over $\phi$. Here, we have set $\hbar=1, \beta=-200$, and $k=0$.

which when entered in (16) leads to a system of differential equations for the coefficients $f_{n}(\phi)$

$$
\begin{gathered}
(1+\phi) f_{0}^{\prime \prime}+2 f_{0}^{\prime}=0, \\
(1+\phi) f_{1}^{\prime \prime}+f_{1}^{\prime}=0, \\
f_{2}^{\prime \prime}=0, \\
(1+\phi) f_{3}^{\prime \prime}+f_{3}^{\prime}=0, \\
(1+\phi) f_{4}^{\prime \prime}-2 f_{4}^{\prime}+36 k(1+\phi) f_{0}=0,
\end{gathered}
$$

which can be solved starting from $f_{0}$

$$
\begin{aligned}
f_{0} & =c_{2}-\frac{c_{1}}{\phi+1}, \\
f_{1} & =c_{3} \log (\phi+1)+c_{4}, \\
f_{2} & =c_{5} \phi+c_{4}, \\
f_{3} & =c_{5}\left(\frac{\phi^{2}}{2}+\phi\right)+c_{6}, \\
f_{4} & =\frac{18 c_{2} k \phi^{2}}{\hbar^{2}}+\frac{36 c_{2} k \phi}{\hbar^{2}}-\frac{18 c_{1} k \phi}{\hbar^{2}}+\frac{c_{6} \phi^{3}}{3}+c_{6} \phi^{2} \\
& +c_{6} \phi+c_{7},
\end{aligned}
$$

The free parameters $c_{n}$ can be fixed according to the boundary conditions on the wave function; to ensure that $\psi(0, \phi)=0$, we must have $c_{1}=c_{2}=0$, and we also set $c_{3}=0$ in order to prevent the presence of the term $\log (1+\phi)$. For the rest of these constants to remain unconstrained, we set $c_{n}=(-1)^{n} / n !$. Thus, the wave function converges to zero at $a=0$. In the same way, one can calculate the same series solution around $a=\infty$, which results in the trivial solution;

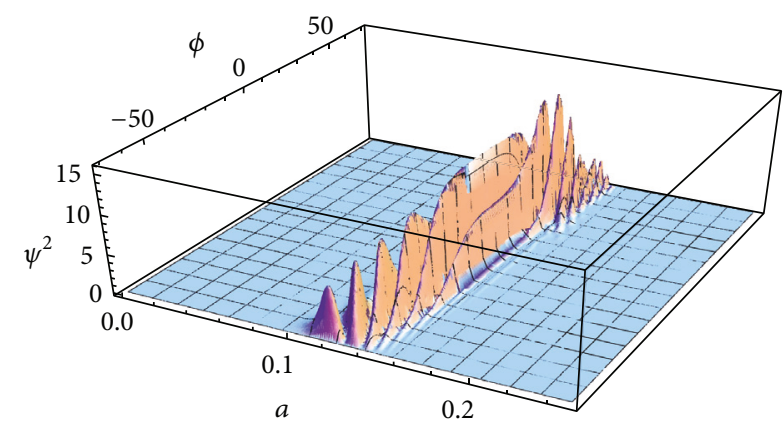

Figure 4: Numerical profile of $\psi(a, \phi)$ with initial conditions: $\psi(0, \phi)=0$ and $\psi\left(a, \phi_{0}\right)=f(a)$, where $\phi_{0}$ defines half the width of our solution domain over $\phi$. Here, we have set $\hbar=1, \beta=-100$, and $k=0$.

all the coefficients are identically zero in this limit. In fact, if we write (17) for $b=1 / a$,

$$
\begin{aligned}
& {\left[\hbar^{2} b^{6}(1+\phi) \frac{\partial^{2}}{\partial \phi^{2}}+\hbar^{2} b^{7} \frac{\partial^{2}}{\partial \phi \partial b}+2 b^{6} \hbar^{2} \frac{\partial}{\partial \phi}\right.} \\
& \left.+36 b^{2} k(1+\phi)+6 \beta \phi^{2}\right] \psi=0,
\end{aligned}
$$

it is consistent with $\psi=0$ at $b=0$. Regarding the $\phi$-direction, we can see that as $\phi \rightarrow \infty$ the $\phi^{2}$ term dominates in (16), forcing the wave function to be zero in this limit.

We have considered values of the parameter $\beta$ for both regimes shown in Figures 1 and 2, and in both cases the wave function has a similar behavior, as seen in Figures 3 and 4 .

\section{Conclusions}

We have studied the classical and quantum formulation of an $f(R)$ modified theory of general relativity based on Starobinsky's model, with a scalar field in a Horndeski type action and in a cosmological setting with a FRW metric. Thus, the Lagrangian and Hamiltonian formulations are straightforward. We consider the numerical solutions for the exact equations in both scenarios, classical and quantum, taking a compact domain for the numerical computation in the second case. Although the dependence of the classical solutions on the parameter $\beta$ can change their topology, the corresponding solutions of the WdW equation do not show such a difference. In fact, for suitable boundary conditions, these solutions tend to zero at the boundaries, pointing to normalizability of the wave function, consistently with the probabilistic interpretation. The form of the wave function suggests that our results could be interpreted by a conditional probability as in [17], where the scalar field plays the role of time.

\section{Conflicts of Interest}

The authors declare that there are no conflicts of interest regarding the publication of this paper. 


\section{References}

[1] A. A. Starobinsky, "A new type of isotropic cosmological models without singularity," Physics Letters B, vol. 91, no. 1, pp. 99-102, 1980.

[2] S. Nojiri and S. D. Odintsov, "Modified gravity with negative and positive powers of curvature: Unification of inflation and cosmic acceleration," Physical Review D, vol. 68, Article ID 123512, 2003.

[3] R. P. Woodard, "Avoiding dark energy with $1 / \mathrm{r}$ modifications of gravity," in Lecture Notes in Physics, vol. 720, p. 403, 2007.

[4] A. De Felice and S. J. Tsujikawa, “ $f(R)$ theories," Living Reviews in Relativity, vol. 13, p. 3, 2010.

[5] T. P. Sotiriou and V. Faraoni, " $f(R)$ theories of gravity," Reviews of Modern Physics, vol. 82, no. 1, article 451, 2010.

[6] J. O'Hanlon, "Intermediate-range gravity: a generally covariant model," Physical Review Letters, vol. 29, no. 2, pp. 137-138, 1972.

[7] D. Langlois and K. Noui, "Degenerate higher derivative theories beyond Horndeski: evading the Ostrogradski instability," Journal of Cosmology and Astroparticle Physics, vol. 2016, no. 2, article no. 034, 2016.

[8] A. Vilenkin, "Classical and quantum cosmology of the Starobinsky inflationary model," Physical Review. D. Particles and Fields. Third Series, vol. 32, no. 10, pp. 2511-2521, 1985.

[9] S. Biswas, A. Shaw, and B. Modak, "Decoherence in the Starobinsky model," General Relativity and Gravitation, vol. 31, no. 7, pp. 1015-1030, 1999.

[10] M. B. Mijić, M. S. Morris, and W. M. Suen, "Initial conditions for $\mathrm{R}+\varepsilon \mathrm{R}^{2}$ cosmology," Physical Review D, vol. 39, no. 6, pp. 1496$1510,1989$.

[11] H. van Elst, J. E. Lidsey, and R. Tavakol, "Quantum cosmology and higher-order Lagrangian theories," Classical and Quantum Gravity, vol. 11, no. 10, pp. 2483-2497, 1994.

[12] M. Kenmoku, K. Otsuki, K. Shigemoto, and K. Uehara, "Classical and quantum solutions and the problem of time in $R^{2}$ cosmology," Classical and Quantum Gravity, vol. 13, no. 7, pp. 1751-1759, 1996.

[13] L. Anderson, E. Aubourg, S. Bailey et al., "The clustering of galaxies in the SDSS-III baryon oscillation spectroscopic survey: baryon acoustic oscillations in the data releases 10 and 11 Galaxy samples," Monthly Notices of the Royal Astronomical Society, vol. 441, pp. 24-62, 2014.

[14] M. V. Ostrogradsky, "Memoires sur les equations differentielles relatives au probleme des isoperimetres," Mémoires de l'Académie impériale des sciences de St. Pétersbourg, vol. 6, p. 385, 1850.

[15] C. G. Knetter, "Effective Lagrangians with higher derivatives and equations of motion," Physical Review D, vol. 49, no. 12, pp. 6709-6719, 1994.

[16] T.-j. Chen, M. Fasiello, E. A. Lim, and A. J. Tolley, "Higher derivative theories with constraints: exorcising Ostrogradsky's ghost," Journal of Cosmology and Astroparticle Physics, vol. 2, 2013.

[17] C. Ramírez and V. Vázquez-Báez, "Quantum supersymmetric FRW cosmology with a scalar field," Physical Review D Particles, Fields, Gravitation and Cosmology, vol. 93, no. 4, Article ID 043505, 2016. 


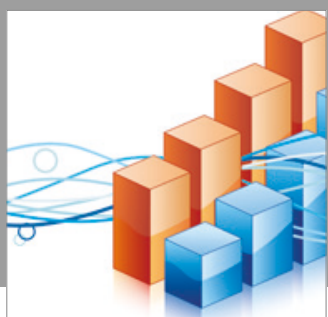

Advances in

Operations Research

vatersals

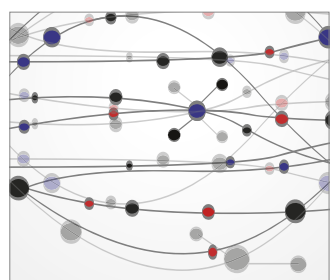

\section{The Scientific} World Journal
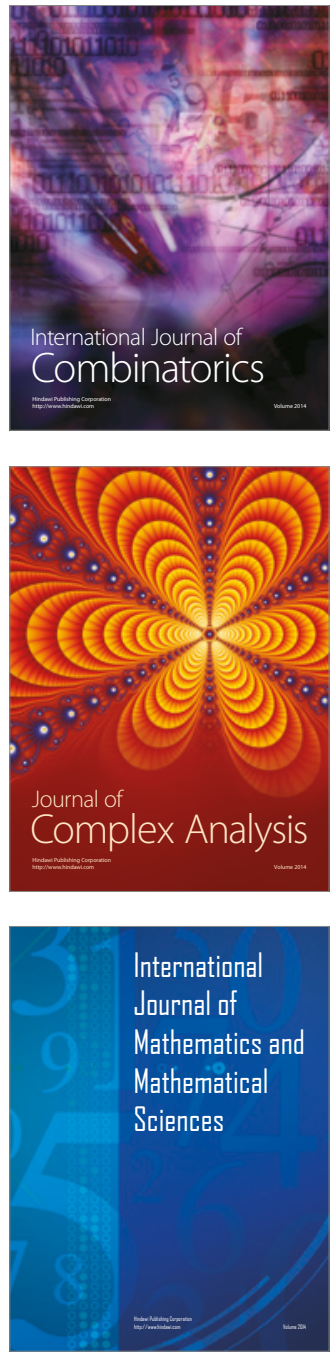
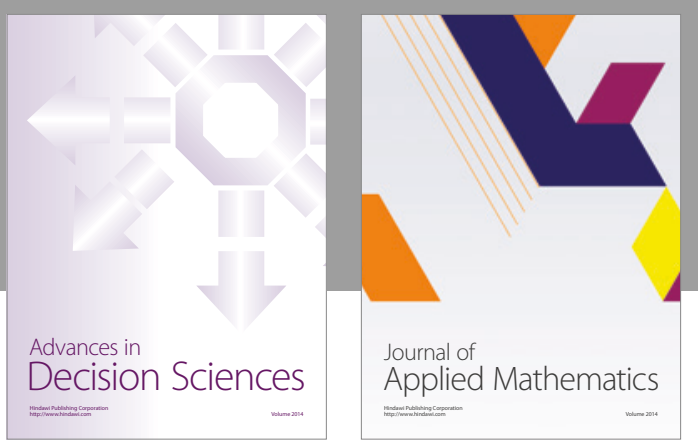

Algebra

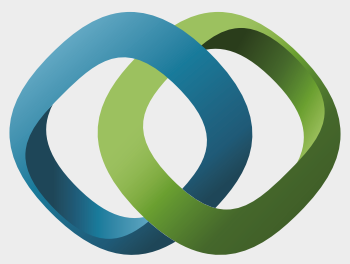

\section{Hindawi}

Submit your manuscripts at

https://www.hindawi.com
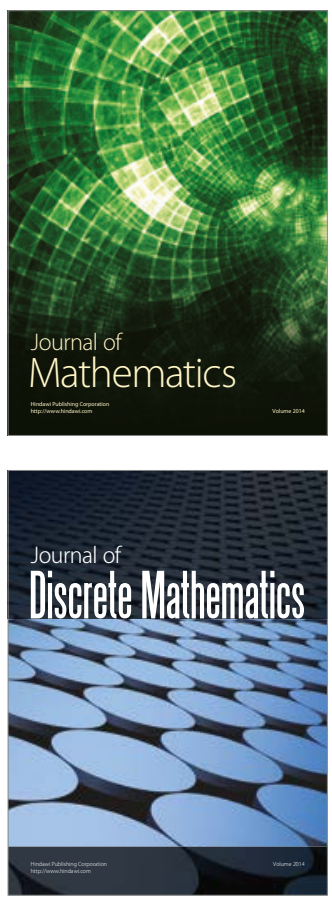

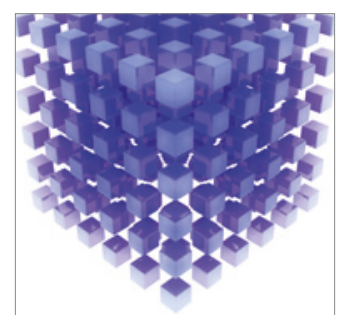

Mathematical Problems in Engineering
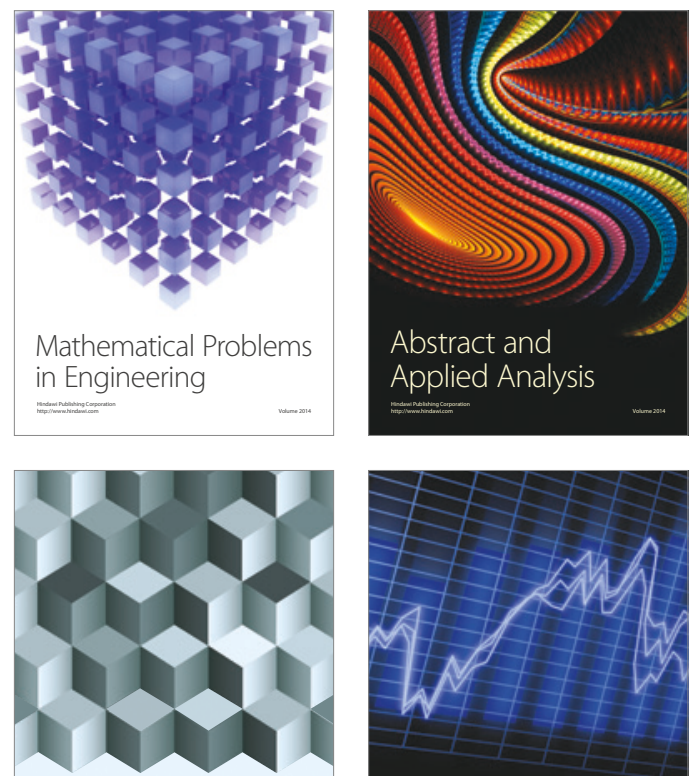

Journal of

Function Spaces

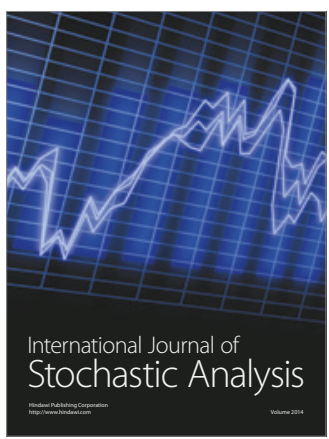

Probability and Statistics
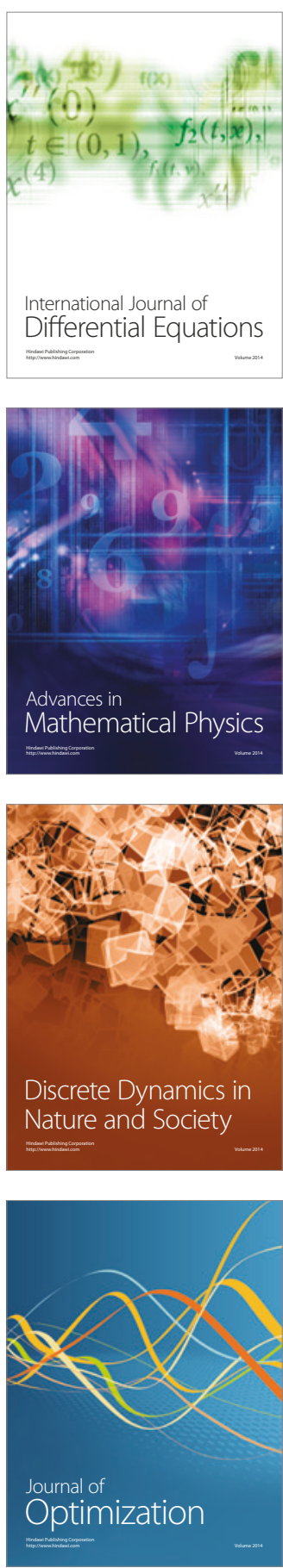